lich für ihre Mandantinnen im Rahmen des Familienrechts ein - und dies spürt man noch bis heute. Außerdem versuchte sie, die Entwicklung des Scheidungsrechts durch Vorträge und den daraus resultierenden inhaltlichen Diskurs vorwärts zu bringen. So lautete die Devise: Wenn man nicht darüber spricht und die Sache anpackt, wird sich niemals etwas ändern. Dieser Diskurs musste allerdings im Rahmen der Anwaltschaft geführt werden, da die meisten Richter zu dieser
Zeit Männer waren, die sich der Thematik des Scheidungsrechts größtenteils nicht widmen wollten.

So veränderten sich im Laufe der Zeit die Gesellschaft, das Scheidungsrecht und die Anzahl der Anwältinnen und Richterinnen. Und es wird sich weiterhin einiges ändern, wenn junge Frauen heutzutage auch der Devise folgen: Man muss die kritischen Themen selbstständig aufgreifen und bearbeiten, um Veränderung zu bewirken.

\title{
Professorin und Mutter: Geht das zusammen?
}

\section{Prof. Dr. Ute Sacksofsky, M.P.A. (Harvard) \\ Goethe-Universität, Frankfurt am Main}

Aufgewachsen bin ich in einer Kleinstadt im südlichen Schwarzwald, die mir in der Pubertät schnell zu klein wurde. So freute ich mich unheimlich auf das Studium - endlich raus. Ich begann mit dem Studium der Mathematik und Biologie für das Lehramt in Freiburg. Ausschlaggebend dafür war wohl die Begeisterung für eine phantastische Lehrerin mit genau dieser Fächerkombination und geringes Vorstellungsvermögen über denkbare Alternativen. Nach einem Semester musste ich aber einsehen, dass ich für Biologie denkbar ungeeignet war: Tiere und Pflanzen interessierten mich nicht wirklich (andere Mitstudierende standen begeistert früh auf, um Vögel zu beobachten - wäre mir nicht im Traum eingefallen), sezieren wollte ich Tiere schon gar nicht und Reagenzgläser tendierten dazu umzufallen, wenn ich in die Nähe kam. Die Mathematik machte mir zwar viel Spaß, aber niemand konnte mir erklären, was ich mit Mathematik einmal beruflich anfangen könnte (Informatik war in Freiburg noch nicht angekommen) außer dem Lehramt und das schied inzwischen aus; dem System Schule einmal entronnen, war mir klar geworden, dass ich dorthin auf keinen Fall zurückkehren wollte. Ein Studienfachwechsel stand an. In meinem ersten Semester hatte ich mir neben Mathematik und Biologie auch einige juristische Veranstaltungen angesehen und war sehr angetan. Jura schien mir die perfekte Verbindung von Logik, die meiner mathematischen Neigung entgegenkam, und Politik, die mich brennend interessierte.

Der Studienfachwechsel wurde mit einem Ortswechsel verbunden. Wegen einer engen Freundin und dem „linken Ruf“ wählte ich Marburg als Kontrastprogramm zum konservativen Freiburg. So kann man sich täuschen. Die Marburger Fakultät war erzkonservativ (z.B. wurde uns untersagt, Flugblätter im Hörsaalgebäude zu verteilen). Hinzu kam, dass viele der Lehrenden einfach schlecht waren. Sie indoktrinierten mit Mittelmaß und Mindermeinung. Nach einem Semester ging ich daher nach Freiburg zurück. Ich begriff schnell, dass mir Vorlesungen mit ihren Massen und dem bloßen Zuhören wenig brachten. Außerdem hatte ich dafür keine Zeit, weil ich mich in der Hochschulpolitik engagierte. Ich war über Jahre im Fachbe- reichsrat, in der Fachschaft und im AStA (bzw. u-asta - wir kämpften gegen die Abschaffung der verfassten Studierendenschaft) aktiv und gründete mit anderen zusammen eine JusoHochschulgruppe. Die einzige Veranstaltungsform, von der ich das Gefühl hatte, richtig zu profitieren, waren Seminare. Mein Studium bestand daher fast ausschließlich aus Seminaren, insgesamt vierzehn - überwiegend im Verfassungsrecht bei den Professoren Böckenförde und Hesse. Diese Seminare waren einfach großartig. Die beiden Professoren in ihrer persönlichen wie theoretischen Unterschiedlichkeit im engen Kreise zu erleben, mit ihnen über die Grundfragen des Verfassungsrechts zu diskutieren, waren echte Highlights. Von beiden habe ich viel gelernt.

Im Studium entdeckte ich auch die Frauenbewegung; mein erstes Buch: „Die Scham ist vorbei“ von Anja Meulenbelt. Wir gründeten eine Juristinnen-Gruppe, lasen zusammen Bücher, diskutierten und demonstrierten. Am Fachbereich gab es keine einzige Professorin, ich kämpfte um die Anrede „Frau“ statt „Fräulein“ (deutlich erschwert dadurch, dass meine Kollegin vom RCDS (Ring Christlich-Demokratischer Studenten) auf der Anrede „Fräulein“ bestand) und um Scheine, die mir nicht als „Herr stud. iur. Ute Sacksofsky“ überreicht wurden (führte zu großem Aufruhr im Fachbereichsrat).

Nach dem ersten Staatsexamen, das ich ohne Repetitor in einer Examensgruppe mit zwei Kommilitonen zusammen vorbereitet hatte, ging ich für zwei Jahre in die USA nach Harvard. Ich hatte ein McCloy-Stipendium erhalten und erwarb den Grad eines Master in Public Administration an der Kennedy School of Government. Die Zeit in den USA war phantastisch. Nie hatte ich mich so frei gefühlt, so viel anderes ausprobiert, so viel Neues kennengelernt und die Zeit einfach genossen. Inhaltlich hatte ich nach dem Ersten Staatsexamen genug von Jura und belegte daher vor allem Kurse in Informatik, Philosophie, in Verwaltungswissenschaften und Ökonomie (wobei mich die Spieltheorie schon wegen ihres Namens begeisterte). Lange stand auf der Kippe, ob ich in den USA bleiben würde (ich hatte mich in das Land und einen Amerikaner verliebt), jedenfalls aber den Referendardienst wollte ich zuerst noch in Deutschland absolvieren.

Zunächst aber stand die Zeit der Promotion an. Ich schwankte heftig zwischen zwei Themen, die mir beide am 
Herzen lagen: „Der Einsatz von artificial-intelligence-Techniken zur juristischen Problemlösung“ und „Das Grundrecht auf Gleichberechtigung“. Dass es zweiteres wurde, ist letztlich Herrn Wassermann zu verdanken. Zufällig fiel mir in den USA das Nachrichtenmagazin „Spiegel“ in die Hände, wonach Herr Wassermann bei der Konferenz der OLG-Präsidenten gesagt hatte, dass man in den Richterdienst nicht streng nach Noten einstellen solle, weil sonst zu viele Frauen in die Justiz gehen würden. Die Empörung darüber gab den Ausschlag. Denn in Amerika war es zu dieser Zeit schon ganz anders. Während ich in Harvard war, durfte ich an einer Gruppe namens „FemCrits“ teilnehmen, insgesamt etwa 15 Juraprofessorinnen aus Cambridge und Boston (und das waren nur die Professorinnen, die sich als Feministinnen begriffen; in Deutschland hatte ich noch nie eine Professorin gesehen). Wir trafen uns einmal monatlich und diskutierten gemeinsam Entwürfe von Aufsätzen der Mitglieder.

Zurück in Deutschland ging ich an das MaxPlanck-Institut für ausländisches öffentliches Recht und Völkerrecht in Heidelberg. Die internationale Atmosphäre mit vielen ausländischen Gästen genoss ich sehr und wir Jüngeren hatten einen engen Kontakt mit vielen stimulierenden Gesprächen und fröhlichen Festen. Dennoch war diese Zeit schwierig. Ich war in eine völlig von Männern dominierte Welt zurückgekehrt; wiederum waren Professorinnen weit und breit nicht zu entdecken und unter den wissenschaftlichen Mitarbeitern waren wir nur ganz wenige Frauen. Mein Promotionsthema stieß bei vielen Männern (glücklicherweise nicht allen!) auf Ablehnung. Die mir Wohlgesonnenen sagten: „Aber Sie, Frau Sacksofsky, Sie hätten doch auch über etwas anderes, i.e. Richtiges, promovieren können"; die mir feindlich Gesonnenen beließen es bei einem verächtlichen „natürlich“. Diese Ablehnung verstärkte die bei mir ohnehin nicht milde ausfallenden Krisen, die wohl jeder bei der Erstellung einer Dissertation durchmacht: Versagensängste, Schreibhemmungen, Verzweiflung. Wenige Wochen vor den Klausuren zum Zweiten Staatsexamen war die Arbeit fertig, im Sommer 1990 das Promotionsverfahren abgeschlossen, kurz darauf war ich Assessorin.

Ich hatte schon lange überlegt, welchen der vielen denkbaren juristischen Berufe ich eigentlich würde ausüben wollen. In der Wahlstation hatte ich ausprobiert, in einer Großkanzlei zu arbeiten, und hatte gelernt, dass dies nichts für mich war: Ich passe mich schlecht in Hierarchien ein und es ist mir zentral, meine Themen wie die Ergebnisse selbst wählen zu können. Das Strafrecht hatte mich nie interessiert, so dass noch Verwaltungsrichterin oder
Ministerialbeamtin zur Wahl standen. Beides konnte ich mir vorstellen, aber die erste Wahl war nachdem die Bewertung meiner Promotion mir den Versuch ermöglicht hatte - die Wissenschaft. Ich hatte herausgefunden, welches die zentralen Kriterien für meinen Beruf waren: möglichst weitgehende Unabhängigkeit und Selbständigkeit - auch (und das ist ganz wichtig) in der Gestaltung meiner Zeit -, finanzielle Sicherheit, die mir Unabhängigkeit ermöglicht, viel Abwechslung und eine Mischung von Ruhe zum Nachdenken und Kontakten zu Menschen; Reichtum ist mir dagegen unwichtig. Nach diesen Kriterien war der Professoren-Beruf der beste (auch wenn die Hochschulpolitiker derzeit alles unternehmen, alle Freiheiten möglichst weitgehend einzuschränken).

\section{Die Lehre kostet enorm viel Energie, mach mir aber weiterhin (meistens) auch viel Spaß.}

Zunächst ging ich als wissenschaftliche Mitarbeiterin an das Bundesverfassungsgericht in Karlsruhe in das Dezernat von Richter Böckenförde (dem Betreuer meiner Doktorarbeit). Eine spannendere Aufgabe war kaum vorstellbar. Ich konnte beobachten (und daran mitwirken), wie Verfassungsrecht in der Praxis gemacht wird und erlebte die Kolleginnen und Kollegen des „Dritten Senates“ als ausgesprochen interessant, klug und angenehm. In dieser Zeit wurde mein Sohn geboren. Da ich in Frankfurt wohnte, vereinbarte ich mit „meinem Richter“, dass ich nach der Rückkehr (Jakob war zu diesem Zeitpunkt ein halbes Jahr alt) nur noch einmal wöchentlich nach Karlsruhe kommen und ansonsten zuhause arbeiten würde. Er war zu einem solchen Entgegenkommen bereit, da ich vor allem ein Großverfahren noch fertigstellen sollte. Jakob war bei einer Tagesmutter, immer abwechselnd bis drei oder bis sechs, was mir sehr entgegenkam, da ich mich an den „langen Arbeitstagen“ auf Jakob freute - und umgekehrt.

Als Jakob knapp zwei Jahre alt war, hörte ich in Karlsruhe auf. Ein Habilitationsstipendium der DFG (Deutsche Forschungsgemeinschaft) ermöglichte es mir, mich ausschließlich auf die Habilitationsschrift (und mein Kind) zu konzentrieren; denn inzwischen war ich alleinerziehend. Diese Phase habe ich als ausgesprochen schwierig in Erinnerung. Mein Leben bestand aus Kleinkind und der Einsamkeit am Schreibtisch - mir fehlte der Kontakt zu Erwachsenen (im Nachhinein würde ich sagen, dass ich damals zu wenig für mich getan habe). Zudem plagten mich Existenzängste. Die Wissenschaft ist 
ein unsicheres Geschäft (bis man eine Professur hat), und ich musste mein Kind und mich ernähren. Bald aber ergab sich eine neue Möglichkeit. Gertrude Lübbe-Wolff holte mich als Mitglied einer Forschungsgruppe zum Thema „Rationale Umweltpolitik - rationales Umweltrecht" für ein Jahr nach Bielefeld ans Zentrum für interdisziplinäre Forschung. Der Umzug in der Schlussphase der Habilitation, neuer Kindergarten, neue Babysitter - nicht ganz einfach, aber mein Sohn machte es prima mit und Jochen Wieland, der Betreuer meiner Habilschrift, unterstützte mich sehr. So verbrachte ich ein Jahr in Bielefeld, ein sehr spannendes Jahr; die interdisziplinäre Arbeit - vor allem mit Ökonomen kam meiner Habilitationsschrift sehr zugute („Umweltschutz durch nicht-steuerliche Abgaben“) und es gelang, die Habilitation in dieser Zeit abzuschließen, wenn auch unter großem Kraftaufwand: Ich begann ein halbes Jahr lang meinen Arbeitstag um vier Uhr morgens, um eine erste Arbeitsphase vor Jakobs Aufwachen hinzukriegen.

\section{Wie ist es als Mutter in der Wissenschaft? \\ Eigentlich vom System immer noch nicht so richtig vorgesehen.}

Und schließlich hatte ich viel Glück: Innerhalb von wenigen Wochen nach meinem Habilvortrag stand ich auf Platz eins der Berufungslisten in Frankfurt und Gießen. Das „Vertreten“ blieb mir ebenso wie eine Zeit der Arbeitslosigkeit erspart; ich konnte im September 1999 meine Professur in Frankfurt antreten. Dort bin ich auch seither geblieben. Frankfurt als Stadt gefällt mir ausgezeichnet (und nicht nur wegen der Nähe des Flughafens): Die Stadt ist international, offen und trotzdem überschaubar. Auch die Universität gefällt mir gut. Es gibt viele Möglichkeiten der interdisziplinären Zusammenarbeit mit Sozial- und Geisteswissenschaftler(inne)n. Institutionalisiert findet das für mich vor allem seinen Ausdruck in der Zusammenarbeit im Rahmen des Cornelia Goethe Centrums für Frauenstudien und die Erforschung der Geschlechterverhältnisse. Und schließlich: Seit auch die juristische Fakultät an den neuen Campus Westend umgezogen ist, haben wir einen wunderschönen Campus.

Die Arbeit als Professorin ist eine Mischung verschiedenster Tätigkeiten. Die Lehre kostet enorm viel Energie, macht mir aber weiterhin (meistens) auch viel Spaß. Auch das Nachdenken am eigenen Schreibtisch, die vielen Kontakte und Diskussionen mit immer wieder neuen Menschen begeistern mich weiter. Weniger erfreulich demgegenüber ist die viele Zeit, die ich mit Korrekturen und Prüfungen verbringe (vielleicht ein Viertel meiner Arbeitszeit), mit Gremienarbeit und Verwaltungstätigkeit. Genossen habe ich schließlich auch meinen Ausflug in die Praxis durch meine fünfjährige Tätigkeit als Landesanwältin beim Hessischen Staatsgerichtshof (im Nebenamt). Die Landesanwaltschaft, eine hessische Besonderheit, wird vom Parlament gewählt, ist aber unabhängig und kann in allen Verfahren vor dem Staatsgerichtshof Stellung nehmen, auch selbst Verfahren einleiten; das waren spannende Jahre. Insgesamt bin ich mit meinem Job sehr zufrieden. Für jemand, die ähnlich „gestrickt“ ist, mit ähnlichen Prioritäten, ist Professorin ein sehr erfüllender Beruf. Es gibt - wie ich mir vorgestellt hatte - viel Abwechslung, viel Möglichkeit der Selbstgestaltung: Ich bin Herrin meiner Zeit und ich bestimme - mit Abstrichen - was ich wann und warum tue.

Wie ist es als Mutter in der Wissenschaft? Eigentlich vom System immer noch nicht so richtig vorgesehen. Hartnäckig hält sich die Vorstellung, dass sich „der Wissenschaftler“ ganz der Wissenschaft widmen und von häuslichen Pflichten freigestellt sein muss. Ein anekdotischer Beleg: Für meine Dissertation bekam ich den Fakultäts-Preis für die beste Promotion. Beim Empfang fragte mich der Dekan, nachdem er mir den Preis überreicht hatte, was ich denn nun machen wollte. Ich: „Ich möchte in die Wissenschaft“. Er: „Sie wollen also keine Kinder.“ Ich: „Doch, eigentlich schon. Haben Sie Kinder?" Er: „Werden Sie doch nicht immer gleich so aggressiv, Frau Sacksofsky. “... Doch auch da verändert sich etwas. Und eigentlich sind die Rahmenbedingungen in der Wissenschaft verhältnismäßig gut. Ein hohes Ausmaß an Flexibilität und Selbstbestimmung in der zeitlichen Gestaltung, die Möglichkeit, viel zuhause zu arbeiten, erleichtern das Leben mit Kind. Aber es ist klar: Die Zeit, die ich mit meinem Kind verbringe, geht von der Arbeitszeit ab; so wird das ein oder andere Buch nicht geschrieben, auch meine Konferenzbesuche schränkte ich sehr ein. Im Rückblick würde ich sagen, dass ich manchmal nicht mutig genug war, zu viel Energie verbrauchte, um mich Systemen anzupassen, die als selbstverständlich voraussetzen, dass eine Hausfrau zuhause den Rücken freihält. Ich kann Sie nur ermuntern, sich die erforderlichen Freiräume zu verschaffen; nach meiner Erfahrung klappt es meist, wenn man es einfordert, während es nicht funktioniert, sich darauf zu verlassen, dass die anderen von sich aus Rücksicht nehmen. In diesem Sinne: Trauen Sie sich! 